\title{
End-to-end test for computed tomography-based high-dose-rate brachytherapy
}

\author{
Fabian Krause, MEng, Franziska Risske, BSc, Susann Bohn, MSc, Marc Delaperriere, MSc, Prof. Jürgen Dunst, MD, PhD, \\ Prof. Frank-André Siebert, PhD \\ Clinic of Radiotherapy, University Hospital Schleswig-Holstein, Kiel, Germany
}

\begin{abstract}
Purpose: One of the important developments in brachytherapy in recent years has been the clinical implementation of complex modern technical procedures. Today, 3D-imaging has become the standard procedure and it is used for contouring and precise position determination and reconstruction of used brachytherapy applicators. Treatment planning is performed on the basis of these imaging methods, followed by data transfer to the afterloading device. Therefore, checking the entire treatment chain is of high importance. In this work, we describe an end-to-end test for computed tomography (CT)-based brachytherapy with an high-dose-rate (HDR) afterloading device, which fulfills the recommendation of the German radiation-protection-commission.

Material and methods: The treatment chain consists of a SOMATOM S64 CT scanner (Siemens Medical), the treatment planning system (TPS) BrachyVision v.13.7 (VMS), which utilizes the calculation formalism TG-43 and the Acuros algorithm v. 1.5.0 (VMS) as well as GammaMedplus HDR afterloader (VMS) using an Ir-192 source. Measurement setups for common brachytherapy applicators are defined in a water phantom, and the required PMMA applicator holders are developed. These setups are scanned with the CT and the data is imported into the TPS. Computed TPS reference dose values for significant points located on the side of the applicator are compared with dose measurements performed with a PinPoint 3D chamber 31016 (PTW Freiburg).

Results: The deviations for the end-to-end test between computed and measured values are shown to be $\leq 5 \%$, when using an implant needle or vaginal cylinder. Furthermore, it can be demonstrated that the test procedure provides reproducible results, while repositioning the applicators without carrying out a new CT-scan.

Conclusions: The end-to-end test presented allows a practice-oriented realization for checking the whole treatment chain for HDR afterloading technique and CT-imaging. The presented phantom seems feasible for performing periodic system checks as well as to verify newly introduced brachytherapy techniques with sufficient accuracy.

J Contemp Brachytherapy 2018; 10, 6: 551-558 DOI: https://doi.org/10.5114/jcb.2018.81026
\end{abstract}

Key words: CT-based brachytherapy, CT planning, end-to-end test, HDR, treatment chain.

\section{Purpose}

Brachytherapy (BT), especially high-dose-rate (HDR) $\mathrm{BT}$, is an important part of modern treatment approaches in radiotherapy. In particular, the possibility of delivering a highly conformal dose distribution on a limited area with high-dose gradient is often used to spare critical structures.

One of the important developments in BT in recent years has been the clinical implementation of complex modern technical procedures for basic treatment concepts. 3D imaging has become the standard procedure, and it is used for contouring and precise position determination as well as reconstruction of used applicators. Treatment planning is performed on the basis of these imaging methods, followed by the transfer of treatment planning data to the afterloading device. Furthermore, model-based dose calculation algorithms (MBDCAs), e.g., using a deterministic solution of the linear Boltzmann transport equation, are available in BT [1]. Despite the fact that BT is a safe treatment method, errors can occur $[2,3]$, especially because BT systems are increasing in complexity. Failures during the applicator reconstruction [4] within the treatment planning system (TPS), based on computed tomography (CT) imaging, have not been detected by using conventional radiographic film checks [5]. Through implementation of MBDCAs and the use of applicators libraries within the TPS, failures caused by imaging errors, misinterpretations, and data processing should be avoided. Therefore, checking the whole treatment planning chain becomes increasingly important for BT procedures. Besides periodical checks, commissioning tests after installation of a new system or system updates
Address for correspondence: Fabian Krause, MEng, Clinic of Radiotherapy, University Hospital Schleswig-Holstein, Karl-Lennert-Krebscentrum Nord, Feldstr. 21, 24105 Kiel, Germany, phone: +49 431500 26522, e-mail: fabian.krause@uksh.de
Received: 09.05 .2018

Accepted: 19.11.2018

Published: 28.12.2018 
should be performed. In accordance with the recommendation of the German radiation-protection-commission [6], an end-to-end test procedure for CT-based BT using an HDR afterloading device is described in this study. In contrast to other system test procedures already published $[7,8,9,10,11,12,13,14,15]$, the presented end-to-end test allows a check of the entire treatment planning chain, including the imaging system. The described system is easy to handle [16], given its compact size. Testing of imaging, applicator reconstruction, data transfer, source strength, dose calculation, and treatment delivery becomes available for clinical routine, with presented procedure.

\section{Material and methods}

\section{Use of end-to-end test}

The treatment planning chain of this study consisted of a SOMATOM S64 single-energy CT scanner by Siemens Medical (Erlangen, Germany), the TPS BrachyVision v.13.7 by Varian Medical Systems (Palo Alto, CA), which utilizes the calculation formalism

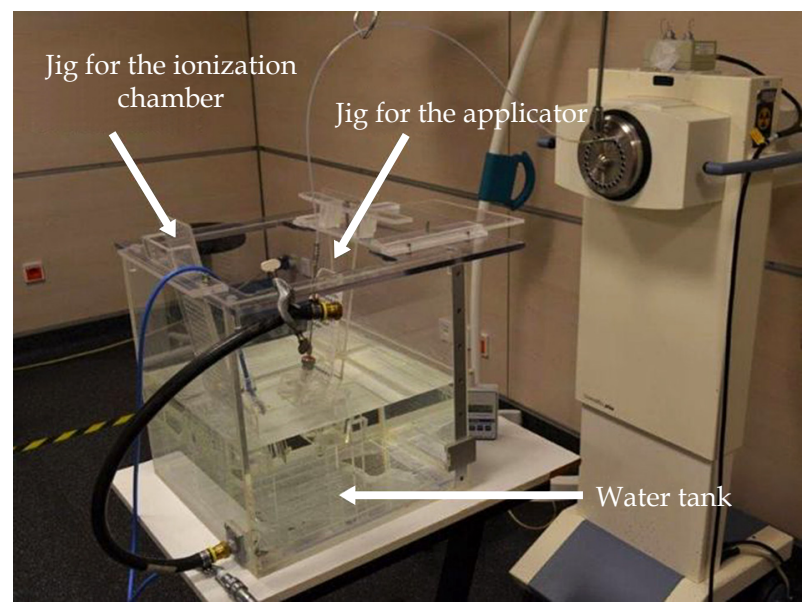

Fig. 1. Phantom setup during measurement with a vaginal cylinder applicator $($ diameter $=2 \mathrm{~cm}$ )

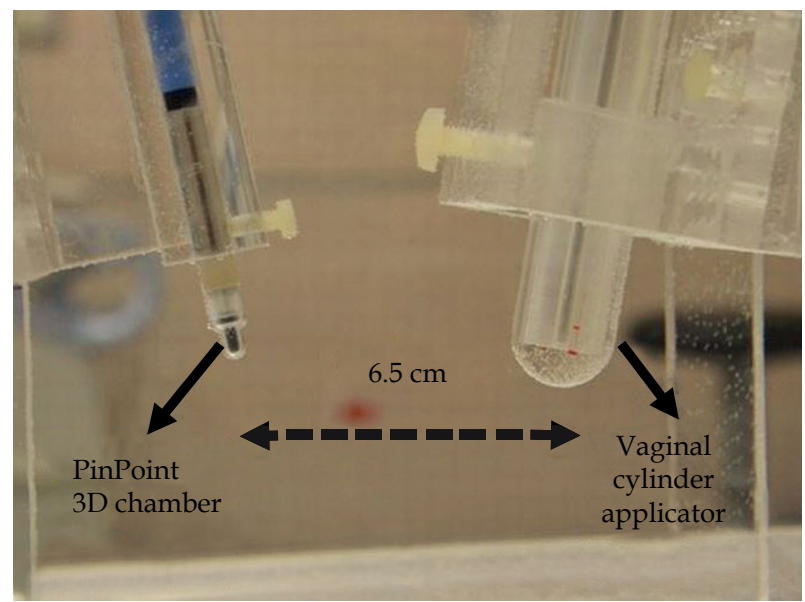

Fig. 2. Detailed view of the used PMMA fixation for the PinPoint 3D chamber as well as vaginal cylinder applicator
TG-43 and the MBDCA Acuros v.1.5.0 (Varian Medical Systems) as well as a GammaMedplus HDR afterloader (Varian Medical Systems) using a nominal $40.700 \mathrm{U}$ ( $\left.1 \mathrm{U}=1 \mathrm{mGy} \cdot \mathrm{m}^{2} \cdot \mathrm{h}^{-1}\right)$ Ir-192 source, and a variety of applicators (steel implant needle, vaginal cylinder, shielded vaginal cylinder). First, several measurement setups for common applicators used in BT were developed with PMMA (polymethyl methacrylate) jigs for a water phantom $(40 \mathrm{~cm} \times 40 \mathrm{~cm} \times 40 \mathrm{~cm})$. These setups were CT scanned using a slice thickness of $0.085 \mathrm{~cm}$, and the data was imported into the TPS. In the TPS, applicators were either reconstructed manually or, if available, imported using applicator library of the TPS. Test plans were then computed in the TPS. Finally, such calculated dose points were compared against measured dose points using a PinPoint 3D chamber (type 31016) of PTW Freiburg (Germany).

\section{The phantom}

One aim of this study was the development of a tissue-equivalent phantom, allowing a suitable procedure for reviewing the treatment planning chain, and using it for consistency checks of CT-based HDR-BT in clinical routine. Accordingly, a water-based phantom with PMMA holders was designed. All dosimetric data were determined in the water phantom specially prepared for this work (Figure 1), which was partly $(\sim 7 \mathrm{~cm}$ above the measurement chamber) filled with water.

Applicator holders as well as measurement chamber holder were custom made PMMA jigs (Figures 2 and 3) mounted in the phantom tank. The PinPoint chamber and the respective applicator were fixed in the water tank at an isotropic distance of at least $7 \mathrm{~cm}$ from the phantom walls to approximate full scatter conditions. The phantom size was kept small because of handling issues. A distance of 7 $\mathrm{cm}$ was sufficient to ensure there are no significant $(<1 \%)$ dose reductions [17] due to lack of full scatter conditions. Thus, the total weight of the water-filled phantom could be limited to $30 \mathrm{~kg}$. To reduce artifacts in the CT imaging,

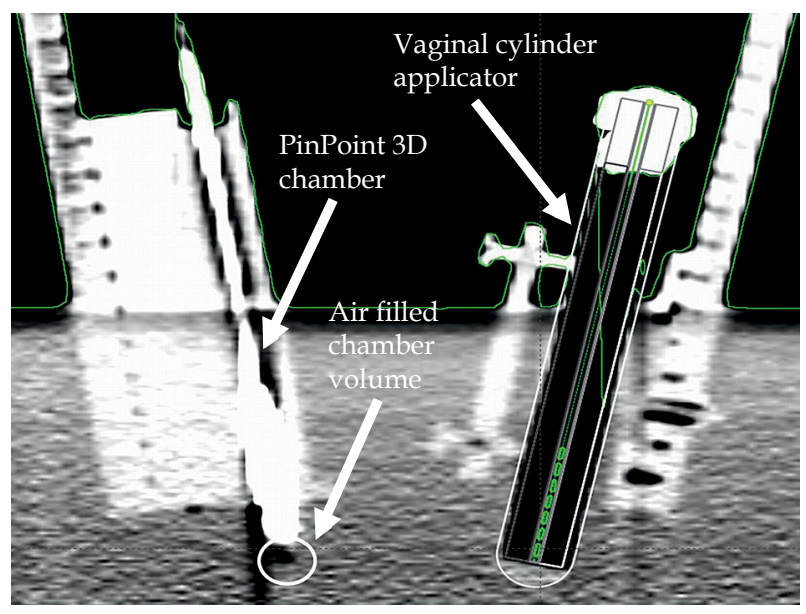

Fig. 3. Close-up sagittal view of the CT data of the used PMMA fixation for the PinPoint 3D chamber and the used unshielded vaginal cylinder (without inlay) taken from the CT scan after applicator reconstruction 
A

B

C

D

E

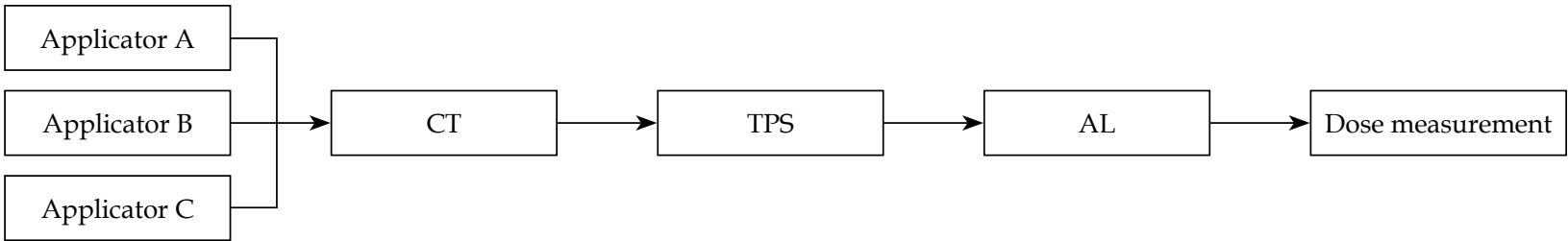

Fig. 4. Schematic workflow: A) selection of an applicator for the phantom; B) run CT scan; C) reconstruction of the used applicator and dose calculation in the TPS; D) data transfer to the afterloading device; E) measurement with PinPoint 3D chamber

any screws required for fixation are manufactured from PMMA or nylon to have only nearly tissue equivalent materials in the phantom setup.

A vaginal cylinder applicator can be used in practice with partial shielding; therefore, some of the PMMA segments can be replaced by tungsten shielding segments to provide a variety of shielding coverage $\left(90^{\circ}\right.$ and $\left.180^{\circ}\right)$. This study uses $180^{\circ}$ shielding. The scan with the vaginal cylinder applicator was carried out without PMMA or tungsten inlay in order to avoid artefacts therefore, having better visibility of the cylinder applicator in the $\mathrm{CT}$ data set. For the applicator reconstruction, a template from the applicator library in the TPS was used, which included all geometry and density information of the used cylinder setting. Consequently, it was not necessary to perform a separate CT scan with the insert to acquire density information of the applicator.

\section{Workflow}

The presented procedure was used to review all planning instances in the treatment planning chain (Figure 4) containing the following components: CT scanner SOMATOM S64, the TPS BrachyVision (v. 13.7), and the afterloading device GammaMedplus. Computed dose values were calculated using the TG-43 formalism and the Acuros $^{\circledR}$ calculation algorithm (Varian Medical Systems, Palo Alto, CA).

\section{Measurement of dose to water $D_{w}$}

All measurements using ionization chambers were performed with a $0.016 \mathrm{~cm}^{3}$ PinPoint 3D chamber 31016 of PTW (Freiburg, Germany). A high spatial resolution was achieved through the small, sensitive volume, which can be used for dose measurements in small photon fields of external beam radiotherapy and also in HDR-BT. Measurement of the dose to water was done according to the DIN 6800-2 [18] report (1):

$D_{w}=\left(M-M_{0}\right) \times N \times k_{T p} \times k_{Q m}$

where $D_{w}$ is dose to water [Gy], $M$ is the measuring signal, $\mathrm{M}_{0}$ is background signal, $\mathrm{N}$ is calibration factor for conversion to dose to water, $\mathrm{k}_{\mathrm{Tp}}$ is correction factor for air density, and $\mathrm{k}_{\mathrm{Qm}}$ is correction factor for radiation quality [19].

The radiation quality correction factor $k_{\mathrm{Q} m}$ was used with reference to Chofor et al. publication [19]. The factor was determined through a semiempirical method by Chofor et al. and for this research, it was summarized to an average value to match the self-made phantom in size as well as the energy of the iridium source. This was done because the phantom dimensions utilized lied within the phantom size range indicated by Chofor et al. For the PinPoint 3D chamber 31016 manufactured by PTW Freiburg, Germany, the correction factor amounts to $k_{\mathrm{Qm}}=1.014$ and corrects all experimentally collected data.

All measured values of the total accumulated dose given in the following sections, corresponded to the mean value of a measurement series consisting of five measurements. The uncertainty was given in standard deviations $(k=1)$.

\section{Applicator-detector distance}

Previous research [20] has shown that a distance of $6 \mathrm{~cm}$ to $8 \mathrm{~cm}$ between the applicator axis and the chamber center was suitable to obtain the largest possible measurement signal, while concurrently considering the steep dose gradient of the Ir-192 source. In this study, a distance of $6.5 \mathrm{~cm}$ from the center of the implant needle or unshielded cylinder (with a diameter of $2 \mathrm{~cm}$ ) to the center of the ion chamber was chosen (Figure 5). A distance of $6 \mathrm{~cm}$ was chosen for the shielded cylinder applicator, considering the dose reduction behind the tungsten shielding.

\section{Functionality}

To verify the functionality of the measurement setup, measured point dose values were compared to the calculated doses. The phantom was sequentially tested with two different applicators: an implantation needle (IN) type $\mathrm{K} 50$ by Varian Medical Systems and an unshielded vaginal cylinder (VC) with a diameter of $2 \mathrm{~cm}$, also by Varian Medical Systems. For both applicators, one dwell position of $60 \mathrm{~s}$ at the applicator's most distal position $(130 \mathrm{~cm})$ of the afterloader was considered.

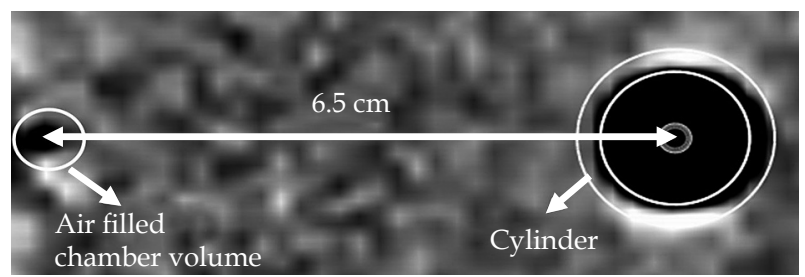

Fig. 5. Frontal view of the PinPoint 3D chamber and the used unshielded vaginal cylinder (without inlay) taken from the CT scan after applicator reconstruction 


\section{Resolution}

To determine the spatial resolution of the phantom setup, the source holding position was gradually shifted in six $0.5 \mathrm{~cm}$ steps, starting from dwell position at $130 \mathrm{~cm}$ up to dwell position at $127.5 \mathrm{~cm}$ within the applicators (IN, VC). Measurements were done at each position. Spatial resolution of the system was evaluated, based on this data.

\section{Reproducibility}

Positioning reproducibility, while assembling and disassembling mechanical parts, was taken into consideration to check the mechanical stability of the phantom. The reproducibility of the measurement setup was investigated by using three reconstructions of the configuration. In order to do so, the measurement setup was demounted and subsequently mounted again in the phantom. The jigs were removed from the water tank and the tank was emptied. Subsequently, the jigs were remounted, and the phantom was refilled. Computed values were determined by means of the CT data set only once at the beginning. This means that only a single CT scan was conducted, and the data derived from it were also used for the rebuilding of the measurement setups. This was done to identify a deviation in the reconstruction of the measurement setup. The dwell position and dwell time considered in 'functionality' were the same as before.

\section{Water level}

Some measurements were repeated at higher fill level ( $\sim 10 \mathrm{~cm}$ above the measurement chamber) and examined

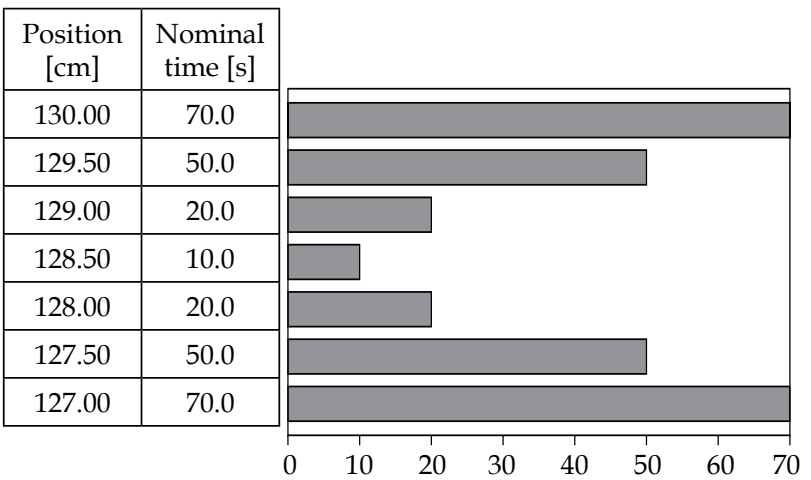

Fig. 6. Dwell times/positions of patient plan for a $180^{\circ}$-shielded vaginal cylinder

Table 1. Dwell times/positions of the test plans

\begin{tabular}{lccc}
$\begin{array}{l}\text { Dwell } \\
\text { position [cm] }\end{array}$ & $\begin{array}{c}\text { Dwell times } \\
\text { plan a [s] }\end{array}$ & $\begin{array}{c}\text { Dwell times } \\
\text { plan b [s] }\end{array}$ & $\begin{array}{c}\text { Dwell times } \\
\text { plan c [s] }\end{array}$ \\
\hline 130.0 & 60 & & 60 \\
\hline 129.5 & 60 & & 20 \\
\hline 129.0 & 20 & 60 & 60 \\
\hline 128.5 & & 60 & \\
\hline 128.0 & & 20 &
\end{tabular}

for deviations in the measured and calculated values to check whether the water fill a level of $7 \mathrm{~cm}$ above the measurement chamber was sufficient with regard to scatter conditions. Two single dose points placed laterally to the Ir-192 source for two single dwell positions of $60 \mathrm{~s}$ at $130 \mathrm{~cm}$ and $127.5 \mathrm{~cm}$ were considered by using an implantation needle.

\section{Planning algorithms}

The commissioning guideline for new modern planning algorithms for BT, just like MBDCAs, is demanding. Therefore, it is worth evaluating how the presented phantom could help medical physicists as a part of the commissioning process to verify differences between dose measurements and calculations. Hence, measurements of a shielded vaginal cylinder were performed behind a $180^{\circ}$-tungsten shielding in the water phantom to point out differences between the calculation formalism TG-43 and the MBDCA Acuros. A patient plan (Figure 6) was computed with both algorithms, and the calculated values were compared with each other as well as against measured values.

\section{Simulation of errors}

Three test plans are defined for the unshielded vaginal cylinder and measured in the phantom. Each plan contains three dwell positions but differs in the arrangement of dwell positions or in the arrangement of dwell times at similar position (Table 1). The total irradiation time was not changed. The dwell position at $130 \mathrm{~cm}$ was located at the distal end of the catheter. This was done to simulate errors caused due to displacement of the applicator (plan b) and to examine to what extent an exchange of the dwell positions can be detected by phantom measurements (plan c).

\section{Results}

\section{Functionality}

The dose deviation between computation and measurement was shown to be $\leq 5 \%$ at the investigated positions (Table 2), when using an implant needle or an unshielded vaginal cylinder for both TG-43 and Acuros computation. Only the dose values for the implantation needle calculated with Acuros showed a slightly higher deviation of $8.5 \%$, which can be attributed to artefacts of the steel needle in the CT scan.

Table 2. Overview of TPS calculated as well as dose points measured laterally to the Ir-192 source

\begin{tabular}{lcccc} 
Applicator & $\begin{array}{c}\text { Distance } \\
\text { applicator- } \\
\text { detector } \\
{[\mathrm{cm}]}\end{array}$ & $\begin{array}{c}\text { TG-43 } \\
{[\mathrm{mGy}]}\end{array}$ & $\begin{array}{c}\text { Acuros } \\
{[\mathrm{mGy}]}\end{array}$ & $\begin{array}{c}\text { Measure- } \\
\text { ment } \\
{[\mathrm{mGy}]}\end{array}$ \\
\hline IN & 6.5 & 167 & 160 & $\begin{array}{c}173 \pm 1 \\
(0.6 \%)\end{array}$ \\
\hline Typ K50 & 6.5 & 202 & 196 & $\begin{array}{c}198 \pm 1.7 \\
(0.9 \%)\end{array}$
\end{tabular}




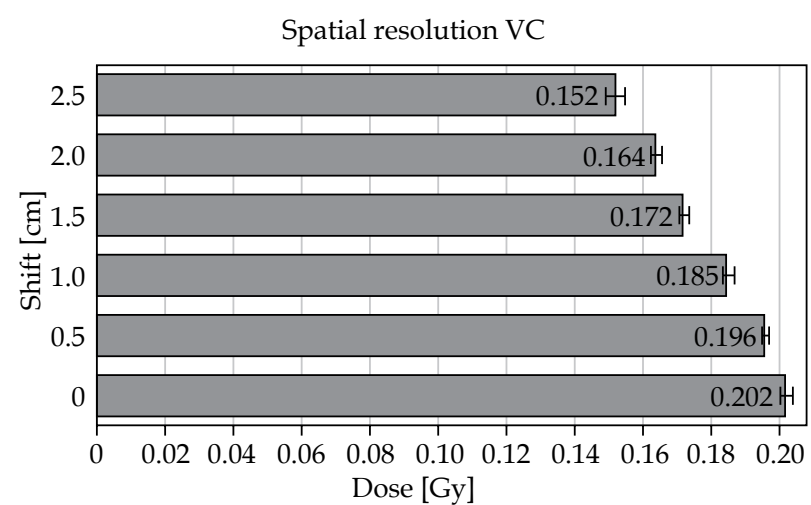

Fig. 7. Dose at vertical shift for an implant needle. Dwell time of $60 \mathrm{~s}$ at every considered holding position

\section{Resolution}

Looking at the experimental results (Figures 7 and 8), it is evident that both applicators have similar characteristics. A shift in dwell positions of $5 \mathrm{~mm}$ or more can be dosimetrically discerned with the presented setup.

\section{Reproducibility}

Furthermore, it can be shown that the system test procedure developed here provides reproducible results, while repositioning the used measurement setup without performing a new CT scan (Tables 3 and 4), considering dose variations of less than $5 \%$ and the resulting small uncertainty of measurement series.

\section{Water level}

Experiments with regard to the effect of the water level of the phantom on the measured water energy dose showed no influence of a water level $>7 \mathrm{~cm}$ above the detector (Table 5). Even at measuring positions with lower water level, because of the vertical shift within the applicator mounted in the water tank (e.g., dwell position $127.5 \mathrm{~cm})$, no dose difference was shown.

\section{Planning algorithms}

It is also possible, as presented here, to determine differences between various calculation methods (e.g., Acuros and TG-43 formalism). In the following, an example was given for a $180^{\circ}$-shielded vaginal cylinder with $2 \mathrm{~cm}$ diameter. As illustrated in Figure 9, the tungsten shielding of the shielded vaginal cylinder can be displayed by

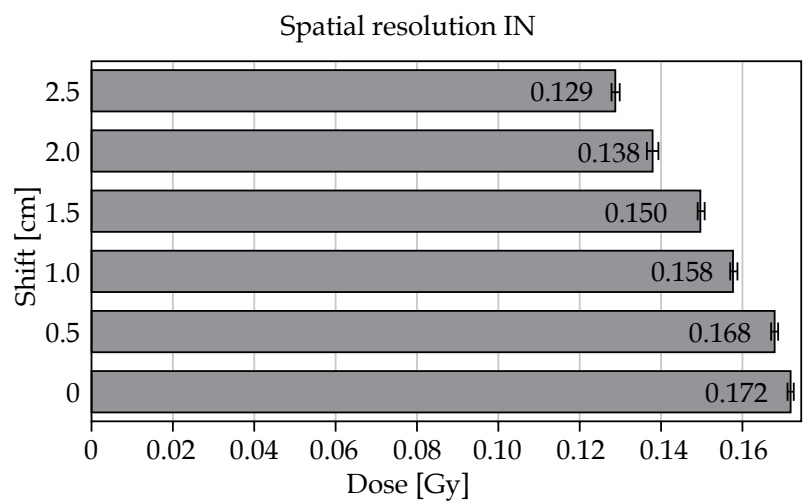

Fig. 8. Dose at vertical shift for an unshielded vaginal cylinder. Dwell time of $60 \mathrm{~s}$ at every holding position considered

calculation with Acuros. In contrast to the isodose levels in Figure 9, in Figure 10, the isodose levels calculated with TG-43 formalism showed no influence of the tungsten shielding.

A comparison shows phantom measurements being in good accordance with the values calculated using Acuros (Table 6). Thus, the system test phantom seems to be suitable to verify the introduction of new techniques like MBDCAs as Acuros.

Table 3. Repositioning of the measurement setup for the implantation needle without a new CT scan

\begin{tabular}{lccc} 
& $\begin{array}{c}\text { Measurement } \\
{[\mathrm{mGy}]}\end{array}$ & $\begin{array}{c}\text { TG-43 } \\
{[\mathrm{mGy}]}\end{array}$ & $\begin{array}{c}\text { Acuros } \\
{[\mathrm{mGy}]}\end{array}$ \\
\hline $\begin{array}{lcc}\text { Repositioning of used } \\
\text { measurement setup } \\
\text { for IN }\end{array}$ & $173 \pm 1(0.6 \%)$ & 167 & 160 \\
& $\frac{}{172 \pm 0.8(0.5 \%)}$ & & \\
\cline { 2 - 2 } & $173 \pm 1(0.6 \%)$ & &
\end{tabular}

Table 4. Repositioning of the measurement setup for the unshielded vaginal cylinder without a new CT scan

\begin{tabular}{|c|c|c|c|}
\hline & $\begin{array}{l}\text { Measurement } \\
{[\mathrm{mGy}]}\end{array}$ & $\begin{array}{l}\text { TG-43 } \\
\text { [mGy] }\end{array}$ & $\begin{array}{l}\text { Acuros } \\
\text { [mGy] }\end{array}$ \\
\hline \multirow{3}{*}{$\begin{array}{l}\text { Repositioning of } \\
\text { used measurement } \\
\text { setup for VC }\end{array}$} & $198 \pm 1.7(0.9 \%)$ & \multirow[t]{3}{*}{202} & \multirow[t]{3}{*}{196} \\
\hline & $202 \pm 1.8(0.9 \%)$ & & \\
\hline & $190 \pm 1.6(0.9 \%)$ & & \\
\hline
\end{tabular}

Table 5. Deviations of the measured and calculated values depending on the fill level of the water phantom, for two different fill levels

Water fill level $7 \mathrm{~cm}$ above the detector

\begin{tabular}{|c|c|c|c|c|}
\hline Position $[\mathrm{cm}]$ & 130.0 & 127.5 & 130.0 & 127.5 \\
\hline Measurement [mGy] & $173 \pm 1(0.6 \%)$ & $129 \pm 1(0.8 \%)$ & $173 \pm 0.9(0.5 \%)$ & $130 \pm 1(0.8 \%)$ \\
\hline TG-43 [mGy] & 167 & 125 & 167 & 125 \\
\hline Acuros [mGy] & 160 & 118 & 160 & 118 \\
\hline
\end{tabular}




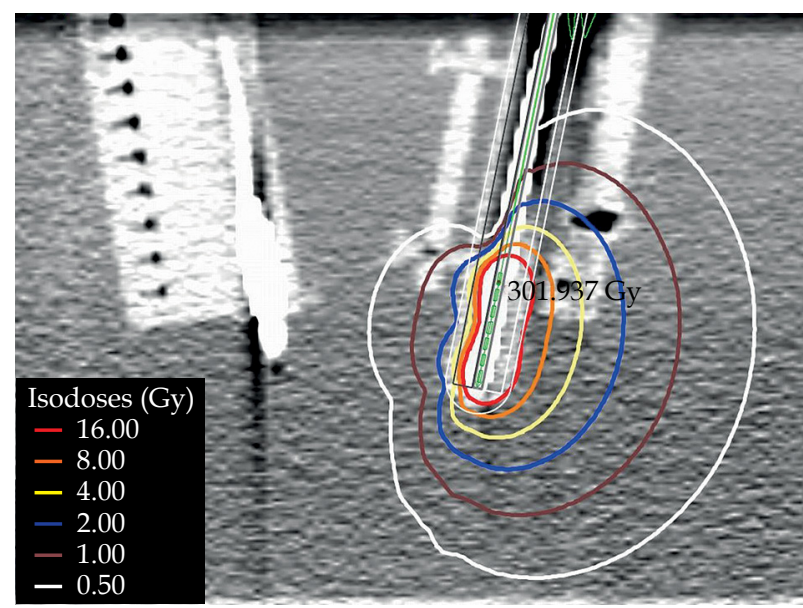

Fig. 9. Patient plan for a $180^{\circ}$-shielded vaginal cylinder calculated with Acuros (dose to medium)

\section{Simulation of errors}

Considering the results of the measured test (plans a-c), a simulated $1 \mathrm{~cm}$ displacement of the detector (plan b)

Table 6. Measurements of a patient plan for a $180^{\circ}$-shielded vaginal cylinder. Total irradiation time of $290 \mathrm{~s}$ at 7 dwell positions with a detector-applicator distance of $6 \mathrm{~cm}$

\begin{tabular}{lccc} 
& $\begin{array}{c}\text { Measurement } \\
{[\mathrm{mGy}]}\end{array}$ & $\begin{array}{c}\text { TG-43 } \\
{[\mathrm{mGy}]}\end{array}$ & $\begin{array}{c}\text { Acuros } \\
{[\mathrm{mGy}]}\end{array}$ \\
\hline $\begin{array}{l}\text { Measuring of } \\
\text { patient plan }\end{array}$ & $185 \pm 0.7(0.4 \%)$ & 865 & 181 \\
for a $180^{\circ}$ - & & & \\
shielded VC & & &
\end{tabular}

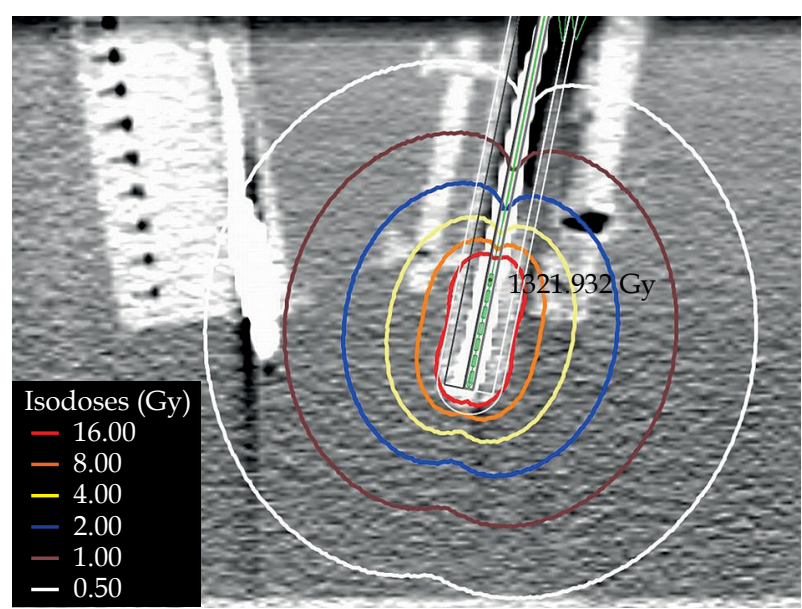

Fig. 10. Patient plan for a $180^{\circ}$-shielded vaginal cylinder calculated with TG-43-formalism

and simulated exchange of dwell positions (plan c) can be identified and measured with the designed phantom.

Due to the applicator-detector distance of $6.5 \mathrm{~cm}$, the dose differences between the original plan a and the simulated switched positions in plan $\mathrm{c}$ at $1.1 \%$, although low, were detectable. A simulated misplacement of $1 \mathrm{~cm}$ in plan b causes a measured dose deviation of $9.4 \%$ at the point of measurement (Table 7).

\section{Uncertainty analysis}

For better understanding of the overall uncertainty of the presented end-to-end test, uncertainties of each individual step of the system test were evaluated (Table 8 ). The first step is the CT-based applicator reconstruction in the TPS. A reconstruction uncertainty of $0.11 \mathrm{~cm}$ (dose

Table 7. Measurement results of error simulation for an unshielded cylinder $(\mathrm{d}=2 \mathrm{~cm})$

\begin{tabular}{lccc} 
& Plan a & Plan b & Plan c \\
\hline Distance applicator-detector $[\mathrm{cm}]$ & 6.5 & 6.5 & 6.5 \\
\hline Measurement $[\mathrm{mGy}]$ & $454 \pm 2.4(0.5 \%)$ & $415 \pm 1.6(0.4 \%)$ & $449 \pm 1.0(0.2 \%)$
\end{tabular}

Table 8. Uncertainty budget for the implant needle and the unshielded vaginal cylinder used within the presented end-to-end test under normal operating conditions

\begin{tabular}{|c|c|c|c|c|}
\hline Procedure & Origin of uncertainty & Type & Uncertainty IN (k = 1) & Uncertainty VC $(\mathrm{k}=1)$ \\
\hline Applicator reconstruction & $\begin{array}{c}\text { Positioning of applicator in } \\
\text { the CT data set }\end{array}$ & A & $3.1 \%$ & $2.5 \%$ \\
\hline Dose calculation & $\begin{array}{c}\text { Calculation of dose in the } \\
\text { TPS }\end{array}$ & $B$ & $2.6 \%$ & $2.6 \%$ \\
\hline \multirow[t]{2}{*}{ Dose delivery } & Source strength & B & $1.5 \%$ & $1.5 \%$ \\
\hline & $\begin{array}{l}\text { Uncertainty of dwell } \\
\text { position }\end{array}$ & $\mathrm{B}$ & $0.6 \%$ & $0.5 \%$ \\
\hline \multirow[t]{3}{*}{ Dosimetry } & $\begin{array}{c}\text { Uncertainty of PinPoint 3D } \\
\text { chamber }\end{array}$ & B & $1.0 \%$ & $1.0 \%$ \\
\hline & Chamber position & A & $0.6 \%$ & $0.5 \%$ \\
\hline & Dose measurement & $A$ & $0.8 \%$ & $0.9 \%$ \\
\hline
\end{tabular}

Combined standard uncertainty IN $(k=1) 4.6 \%$; combined standard uncertainty VC $(k=1) 4.2 \%$ 
effect $3.1 \%$ ) for the implant needle and $0.07 \mathrm{~cm}$ (dose effect $2.5 \%$ ) for the vaginal cylinder were found. The uncertainty for the dose calculation was assumed to be $2.6 \%$ $[21,22]$ and for the source strength - 1.5\% [21]. Furthermore, a systematic error of $0.01 \mathrm{~cm}$ was estimated for the uncertainty of the dwell positions [22]. This resulted in a dose effect of $0.6 \%$ for the implant needle and $0.5 \%$ for the vaginal cylinder. The long-term stability of the ionization chamber used was given as $1 \%$. The uncertainty of the measurement chamber position can be determined at $0.01 \mathrm{~cm}$, which resulted in dose effects of $0.6 \%$ for the implant needle and $0.5 \%$ for the vaginal cylinder. By repeating the dose measurements five times, the uncertainty for the dose measurement process was determined at $0.8 \%$ (implant needle) and $0.9 \%$ (vaginal cylinder). Thus, an overall uncertainty of $4.6 \%$ for the implant needle and $4.2 \%$ for the vaginal cylinder was calculated using a simple quadrature sum of the individual procedures, as shown in Table 8 . The results for the vaginal cylinder can be adapted for the shielded vaginal cylinder in the same way.

\section{Discussion}

Several approaches to determine the accuracy of dwell positions and dwell times for HDR-BT have been published in the last 10 years. A wide variety of detectors such as film dosimetry [7], ion chamber array [8,9], luminescence dosimetry $[10,11]$ as well as diode array $[12,13]$ test procedures deliver higher accuracy regarding resolution of dwell positions and dwell times. However, none of these test procedures measures in water surrounding or is suitable for use as an end-to-end test for the whole treatment planning chain. Also, real-time dosimetry $[23,24,25]$ is available for in vivo dosimetry in BT. Though real-time measurements can identify a variety of different error scenarios (e.g., applicator displacement, interchanged guide tubes during therapy) and are a good indicator for the stability of the system, they are not suitable to exactly locate where errors occur during image and data processing (such as during the image data transfer into the planning system). Furthermore, the equipment required is expensive and not widely available. There are few concepts published in the literature related to endto-end tests in HDR-BT. The published studies either focus only on displacement effects [14], or while delivering more precise results with regard to the combined standard uncertainty (3.2\%) [16], which due to the size and construction of the used phantoms are not suitable for periodic in-house test procedures to check the entire treatment planning chain in clinical practice. Another system check shown [15], based on a PMMA-phantom, provides a system check for the planning chain without inclusion of the imaging system. Hence, the presented system check procedure in our opinion bridges a gap, because it not only allows a check of the entire planning chain with a dosimetric verification in water medium, but by reason of its size, it is also easy to handle and more flexible for testing a variety of different applicators with the same phantom as in other published concepts. Although PMMA-based phantoms provide a fixed geome- try [15], an advantage of the presented water phantom is the possibility of easy adaption for checking a variety of other applicators. Instead of drilling new channels within a PMMA block, only minor modification of the applicator jig is necessary. Furthermore, for measurements within a water filled phantom, no additional correction factor for PMMA has to be applied. Moreover, due to their composition, the homogeneity of commercial plastic phantom materials is not always reproducible [26]. Thus, possible failure through the use of a wrong conversion factor is avoided. Moreover, the phantom presented in this study can be developed at a low cost and from parts available in most clinics as standard equipment. The phantom in this study can also be used to complement the commissioning of MBDCAs according to the TG-186 report $[1,20]$.

\section{Conclusions}

The system test procedure presented in this study provides a practice-oriented realization for checking the entire treatment planning chain for HDR afterloading technique and CT imaging. It seems feasible to perform periodic system tests as well as to control the introduction of new techniques with sufficient accuracy. The dosimetric errors for the measured dose according to the AAPM Task Group No. 138 and GEC-ESTRO report [21] for high energies $(3 \%$ with $\mathrm{k}=1)$ are for the applicator detector distance of $6 \mathrm{~cm}$ and $6.5 \mathrm{~cm}$, respectively, clearly undercut (about $2 \%$ with $\mathrm{k}=1$ ). With regard to the uncertainties (IN 5\%, VC 8\%) to be adopted in HDR-BT, as published in the directives by the GEC-ESTRO and AAPM [22], a sufficient accuracy is achieved. Thus, it is possible to check several different error scenarios during the planning process (e.g., dose calculation, source positioning, applicator reconstruction, data transfer) while using this phantom. Furthermore, the phantom can be used to verify new clinical approaches such as new planning algorithms (e.g., as recommended in the TG-186 report [1]). As part of a periodic test procedure, it is also possible to use the phantom for constancy checks of the entire treatment planning chain in clinical practice. Analysis of the resulting measurement uncertainties has shown that an important uncertainty factor is the measuring system or rather the phantom itself. It has, therefore, to be further elaborated how far the constructional parts of the phantom can be improved, e.g., by a CNC machine for manufacturing critical parts or using a smaller detector (e.g., microDiamond type 60019 of PTW Freiburg, Germany).

\section{Disclosure}

Authors report no conflict of interest.

\section{References}

1. Beaulieu L, Carlsson TA, Carrier JF et al. Report of the Task Group 186 on model-based dose calculation methods in brachytherapy beyond the TG-43 formalism: Current status and recommendations for clinical implementation. Med Phys 2012; 39: 6208-6236.

2. Wilkinson DA, Kolar MD. Failure modes and effects analysis applied to high-dose-rate brachytherapy treatment planning. Brachytherapy 2013; 12: 382-386. 
3. Mayadev J, Dieterich S, Harse R et al. A failure modes and effects analysis study for gynecologic high-dose-rate brachytherapy. Brachytherapy 2015; 14: 866-875.

4. Roué A, Ferreira IH, Van Dam J et al. The EQUAL-ESTRO audit on geometric reconstruction techniques in brachytherapy. Radiother Oncol 2006; 78: 78-83.

5. Okamoto H, Nakamura S, Nishioka $S$ et al. Independent assessment of source position for gynecological applicator in high-dose-rate brachytherapy. J Contemp Brachytherapy 2017; 9: 477-486.

6. Strahlenschutzkommission. Physikalisch-technische Qualitätssicherung in der Strahlentherapie - Vorschläge zur Prüfung des gesamten Behandlungssystems. Empfehlung der Strahlenschutzkommission. BANZ 2011; 66: 1563.

7. Rickey DW, Sasaki D, Bews J. A quality assurance tool for high-dose-rate brachytherapy. Med Phys 2010; 37: 2525-2532.

8. Manikandan A, Biplab S, David PA et al. Relative dosimetrical verification in high dose rate brachytherapy using two-dimensional detector array IMatriXX. J Med Phys 2011; 36: 171-175.

9. Gainey M, Kollefrath M, Bruggmoser G. SU - E - T - 150: Brachytherapy QA Employing a High Resolution Liquid Filled Ionisation Chamber Array: Initial Experience and Limitations. Med Phys 2015; 42: 3366.

10. Casey KE, Alvarez P, Kry SF et al. Development and implementation of a remote audit tool for high dose rate (HDR) Ir-192 brachytherapy using optically stimulated luminescene dosimetry. Med Phys 2013; 40: 112102.

11. Guiral P, Ribouton P, Jalade R et al. Design and testing of a phantom and instrumented gynecological applicator based on GaN dosimeter for use in high dose rate brachytherapy quality assurance. Med Phys 2016; 43: 5240-5249.

12. Espinoza A, Beeksma B, Petasecca M et al. The feasibility study and characterization of a two-dimensional diode array in "magic phantom" for high dose rate brachytherapy quality assurance. Med Phys 2013; 40: 111702.

13. Espinoza A, Petasecca M, Fuduli I et al. The evaluation of a 2D diode array in "magic phantom" for use in high dose rate brachytherapy pretreatment quality assurance. Med Phys 2015; 42: 663-673.

14. Gao W. Minimizing targeting error in high-dose-rate brachytherapy with an end-to-end source positioning test. Brachytherapy 2016; 15: 171-172.

15. Kollefrath M, Bruggmoser G, Nanko N et al. In-phantom dosimetric measurements as quality control for brachytherapy: System check and constancy check. Z Med Phys 2015; 25: 176185.

16. Palmer AL, Lee C, Ratcliffe AJ et al. Design and implementation of a film dosimetry audit tool for comparison of planned and delivered dose distribution in high dose rate (HDR) brachytherapy. Phys Med Biol 2013; 58: 6623-6640.

17. Dössel O, Schlegel WC (eds.). Effect of Varying Phantom Size in Dosimetry of Iridium-192: A Comparison of Experimental Data with EGSnrc Monte Carlo Calculation. Proceedings of the World Congress on Medical Physics and Biomedical Engineering, 2009 September 7-12; Munich, Germany. Springer 2009; Berlin, Heidelberg.

18. Deutsches Institut für Normung. Dosismessverfahren nach der Sondenmethode für Photonen- und Elektronenstrahlung - Teil 2: Dosimetrie hochenergetischer Photonen- und Elektronenstrahlung mit Ionisationskammern. Norm DIN 6800-2 2008. Beuth; Berlin 2008.

19. Chofor N, Harder D, Selbach HJ, Poppe B. The mean photon energy $\overline{E F}$ at the point of measurement determines the detector-specific radiation quality correction factor $\mathrm{kQ}, \mathrm{M}$ in (192) Ir brachytherapy dosimetry. Z Med Phys 2016; 26: 238-250.
20. Krause F, Krause TM, Siebert FA. Commissioning of a model-based dose calculation algorithm for brachytherapy according to the TG-186 report. Radiother Oncol 2015; 115: 140.

21. DeWerd LA, Ibbott GS, Meigooni AS et al. A dosimetric uncertainty for photon-emitting brachytherapy sources: Report of AAPM Task Group No. 138 and GEC-ESTRO. Med Phys 2011; 38: 782-801.

22. Kirisits C, Rivard MJ, Baltas D et al. Review of clinical brachytherapy uncertainties: Analysis guidelines of GEC-ESTRO and the AAPM. Radiother Oncol 2014; 110: 199-212.

23. Andersen CE, Nielsen SK, Lindegaard JC et al. Time-resolved in vivo luminescence dosimetry for online error detection in pulsed dose-rate brachytherapy. Med Phys 2009; 36: 5033-5043.

24. Kertzscher G, Andersen CE, Siebert FA et al. Identifying afterloading PDR and HDR brachytherapy errors using real-time fiber-coupled Al2O3: C dosimetry and a novel statistical error decision criterion. Radiother Oncol 2011; 100: 456-462.

25. Tanderup K, Beddar S, Andersen CE et al. In vivo dosimetry in brachytherapy. Med Phys 2013; 40: 070902.

26. Seuntjens J, Olivares M, Evans M et al. Absorbed dose to water reference dosimetry using solid phantoms in the context of absorbed-dose protocols. Med Phys 2005; 32: 2945-2953. 